\title{
Modeling the longitudinal outcomes of congestive heart failure patients: A case study at Wachemo University Nigist Eleni Mohammed Memorial Referral Hospital
}

Mohammed Sultan ( $\sim$ sutankarebo@gmail.com )

Wachemo University

Ritbano Ahmed

Wachemo University

\section{Research Article}

Keywords: Joint modeling, linear mixed model, congestive heart failure

Posted Date: June 8th, 2021

DOI: https://doi.org/10.21203/rs.3.rs-601836/v1

License: (c) (1) This work is licensed under a Creative Commons Attribution 4.0 International License.

Read Full License 


\section{Modeling the longitudinal outcomes of congestive heart failure patients: A case study at Wachemo University Nigist Eleni Mohammed Memorial Referral Hospital}

Mohammed Sultan ${ }^{1}$ and Ritbano Ahmed Abdo ${ }^{2}$

${ }^{1}$ Department of Statistics, Collage of Natural and Computational Science, Wachemo University, Hosanna, Ethiopia

${ }^{2}$ Department of Midwifery, College of Medicine and Health Sciences, Wachemo University, Hossana, Ethiopia

*Corresponding author

Email:sultankarebo@gmail.com 


\section{Abstract}

The linear mixed model is one of the common models used to analyze the longitudinal data; it may comprise of Separate (Univariate), joint Bivariate or joint multivariate linear mixed model, which is predicated on the number of response variables incorporated in the analysis. Adjusting for correlation matrix and covariance matrix between and within subjects is one reason why modern longitudinal data analysis techniques are deemed more appropriate than some of the previous methods of analysis. Some studies assume that the correlation between observations is zero. However, it is unlikely that repeated measurements on the same individual will actually be independent. To that end, comparing the different linear mixed models identifying the appropriate model demonstrates that the evolution of patients with congestive heart failure is necessary.

In this study the separate, bivariate and multivariate linear mixed models were compared with different covariance and correlation structures. Finally, a multivariate linear mixed model with autoregressive order one correlation structure and unstructured covariance structure for random effects, to consider within and between patients' variations, was considered as the best model to depict the evolution of patients with congestive heart failure. 


\section{Background}

In longitudinal data, the dependent variable is measured several times, whereas the individual subject is used as a random factor. More specifically, Linear mixed models (LMMs) or General Linear mixed models (GLMM) can be used to account for repeated measures in longitudinal studies as well as for random effects [1].

Mixed models are commonly used to tackle correlated data or hierarchical data in the fields of health and social sciences [1]. In this context, the linear mixed model is one of the mixed models that fit the mean response as a combination of population characteristics (fixed effects) that are assumed to be shared by all individuals and subject-specific effects (random effects) unique to a particular individual [2]. Depending on the number of response variables incorporated into the analysis, there are different types of linear mixed models: separate (univariate), joint bivariate and joint multivariate linear mixed models.

The separate univariate linear mixed model clarifies the relationship between a continuous response variable and the independent variables, with both fixed and random effects. Conversely, the (bivariate or multivariate) linear mixed model describes the relationship between two or more continuous response variables and the independent variables [3]. In most longitudinal experiments, the number of outcomes measured repeatedly in the subjects is found to exceed one. However, whenever the purpose is a comparison of longitudinal trends between outcomes, or the purpose is to identify the association between the outcomes and how that association evolves over time, a joint analysis of all outcomes is required [4].

In a different study, analysing the multiple outcomes jointly is considered the best model. For example, multivariate linear mixed models were proposed to analyse multiple outcomes as well as to assess and test for a global exposure effect across outcomes, while assuming a flexible 
correlation structure for the multiple outcomes [5]. A study conducted in Spain revealed that the multivariate approach performs better than the univariate approach [6]. In other studies conducted in France, the bivariate random effects model was significantly better than two separate univariate random effects models $[7,8]$.

The correlation matrix and/or covariance matrixbetween observations also plays an important role in the analysis of longitudinal data. Adjusting for correlation between observations is one reason why modern longitudinal data analysis techniques are more appropriate than some of the previous analysis methods [9]. Correlation structures are used to model dependency among within-group errors $[10,11]$. Some studies assume that the correlation between measurement errors amounts to zero [12]. This assumption may not hold in some data situations, especially if the time between observations is very short (i.e. daily or weekly observations) or if the correlation between observations does not decrease very quickly [13]. It is unlikely that repeated measurements on the same individual will actually be independent [9]. Ignoring the existing correlation of longitudinal data may lead to incorrect and inefficient inferences, and it may increase the risk of Type I errors and of underestimating standard errors [9]. Consideration of all these issues is necessary in order to select an appropriate model with the mean, covariance and correlation structure for the longitudinal data of Congestive Heart Failure patients $(\mathrm{CHF})$ patients. 


\section{Methods}

\section{Source of data and Variables}

The current study's data comprises 154 adult patients suffering from congestive heart failure, who reported that they had visited a hospital at least thrice within two years. The patients' pulse rate, respiratory rate and weight were considered as response variables, while age, sex, time, place of residence, New York Heart Association Class, diagnostic history, left ventricle ejection fraction, valvular heart disease, smoking status, diabetes status, diastolic blood pressure and systolic blood pressure were deemed as independent variables.

\section{Statistical Methods of Data Analysis}

The model which used in this study was a linear mixed effect model which contains fixed effects, random effects and measurement errors and are referred as $\beta, b_{i}$, and $\varepsilon_{i}$ respectively. The three endpoints are longitudinally measured as a vector of responses, $Y_{i}(t)$ at each occasion with this model:

$Y_{i}(t)=X_{i}(t)^{T} \beta+Z_{i}(t)^{T} b_{i}+\varepsilon_{i}(\mathrm{t})$

Where $\varepsilon_{i}=\left[\varepsilon_{i}\left(t_{1}\right), \varepsilon_{i}\left(t_{2}\right), \varepsilon_{i}\left(t_{3}\right)\right]^{T} \sim \operatorname{MVN}\left(0, R_{i}\right), b_{i} \sim \operatorname{MVN}(0, \mathrm{D}), \operatorname{Cov}\left(b_{i}, \varepsilon_{i}\right)=0$, D is the variance-covariance matrix of random effects. $R_{i}=I_{n_{i}} \otimes \sum_{3 X 3}$ is the variance covariance matrix of 3 endpoints (symptoms)

Let $Y_{i}=\left[\begin{array}{l}Y_{1 i}(t) \\ Y_{2 i}(t) \\ Y_{3 i}(t)\end{array}\right]$, the response vectors for the subject $\mathrm{i}$ with $Y_{\mathrm{ki}}$, the $n_{\mathrm{ki}}$ vector of the end points $\mathrm{k}(\mathrm{k}=1,2,3)$ with $n_{1 i}=n_{2 i}=n_{3 i}=n_{i}$ so model for multivariate longitudinal Gaussian data is: $\left.\begin{array}{rl}Y_{1 i}(t) & =\mu_{1}(\mathrm{t})+\alpha_{1 i}+b_{1 i}(t)+\varepsilon_{1 i}(\mathrm{t}) \\ Y_{2 i}(t) & =\mu_{2}(\mathrm{t})+\alpha_{2 i}+b_{2 i}(t)+\varepsilon_{2 i}(\mathrm{t}) \\ Y_{3 i}(t) & =\mu_{3}(\mathrm{t})+\alpha_{3 i}+b_{3 i}(t)+\varepsilon_{3 i}(\mathrm{t})\end{array}\right\}$ 
$\left., \mathrm{b}=\left(\begin{array}{l}\alpha_{i} \\ b_{i}\end{array}\right)=\left(\begin{array}{l}\alpha_{1 i} \\ \alpha_{2 i} \\ \alpha_{3 i}\end{array}\right),\left(\begin{array}{l}b_{1 i} \\ b_{2 i} \\ b_{3 i}\end{array}\right)\right), \quad \mathrm{N}\left(0, D_{6 \times 6}\right)$, where $\mathrm{D}$ is the variance-covariance matrix for the 3 intercepts and 3 slopes associated with every outcome (covariance matrix for random effects). $\left(\varepsilon_{1 i}(\mathrm{t}), \varepsilon_{2 i}(\mathrm{t}), \varepsilon_{3 i}(\mathrm{t})\right) \sim \mathrm{N}\left(0, \sum_{3 X 3}\right)$, for all $\mathrm{t}$ Where $\sum_{3 X 3}$ is the variance covariance matrix of 3 endpoints (symptoms) or (covariance matrix for error components). The variance-covariance matrixes used in this study were listed in the table 1. 
Table 1 The Variance-Covariance/Correlation Structures

\begin{tabular}{|c|c|c|c|}
\hline Type & Matrix R & & Number of Parameters \\
\hline Unstructured (UN) & $\mathrm{UN}=\left[\begin{array}{cccc}\sigma_{1}{ }^{2} & \sigma_{12} & \ldots & \sigma_{1 i} \\
\sigma_{21} & \sigma_{2}{ }^{2} & \ldots & \sigma_{2 i} \\
: & : & : & : \\
\sigma_{i 1} & \sigma_{i 2} & \ldots & \sigma_{i}{ }^{2}\end{array}\right]$ & & $\mathrm{i}(\mathrm{i}+1) / 2$ \\
\hline Compound Symmetry (CS) & $\mathrm{CS}=\sigma^{2}\left[\begin{array}{cccc}1 & \rho & \cdots & \rho \\
& 1 & \cdots & \vdots \\
& & \ddots & \rho \\
& & & 1\end{array}\right]$ & & 2 \\
\hline $\begin{array}{l}\text { Heterogeneous Compound } \\
\text { symmetry (CSH) }\end{array}$ & $\mathrm{CSH}=\left[\begin{array}{ccc}\sigma_{1}{ }^{2} & \sigma_{1} \sigma_{2} \rho & \ldots \\
& \sigma_{2}{ }^{2} & \ldots \\
& & \ddots\end{array}\right.$ & $\left.\begin{array}{c}\sigma_{1} \sigma_{i} \rho \\
\sigma_{2} \sigma_{i} \rho \\
\vdots \\
{\sigma_{i}^{2}}^{2}\end{array}\right]$ & $\mathrm{i}+1$ \\
\hline $\begin{array}{l}\text { First Order Autoregressive } \\
(\operatorname{AR}(1))\end{array}$ & $\operatorname{AR}(1)=\sigma^{2}\left[\begin{array}{ccc}1 & \rho & \ldots \\
& 1 & \ldots \\
& & \ddots\end{array}\right.$ & $\left.\begin{array}{c}\rho^{i-1} \\
\rho^{i-2} \\
\vdots \\
1\end{array}\right]$ & 2 \\
\hline Toeplitz (TOEP) & $\mathrm{TOEP}=\left[\begin{array}{ccc}\sigma^{2} & \sigma_{12} & \sigma_{13} \\
& \sigma^{2} & \sigma_{12} \\
& & \sigma^{2}\end{array}\right.$ & $\left.\begin{array}{cc}\cdots & \sigma_{1 i} \\
\cdots & \sigma_{1 i-1} \\
\cdots & \vdots \\
\ddots & \sigma_{12} \\
& \sigma^{2}\end{array}\right]$ & $\mathrm{T}$ \\
\hline $\begin{array}{l}\text { Variance } \\
\text { (VC) }\end{array}$ & $\mathrm{VC}=\left[\begin{array}{cccc}\sigma_{1}{ }^{2} & 0 & \ldots & 0 \\
0 & \sigma_{2}{ }^{2} & \ldots & 0 \\
: & : & : & : \\
0 & 0 & \ldots & \sigma_{i}{ }^{2}\end{array}\right]$ & & $\mathrm{Q}$ \\
\hline
\end{tabular}

To estimate the parameters pertaining to the model used, both maximum likelihood estimation (MLE) and restricted maximum likelihood estimation were performed. The ML method primarily maximizes the log likelihood with respect to the variance parameters, while treating the fixed effects parameters $\beta$ as constant. After determining the variance parameter estimates, the fixed effects parameters are identified by ascertaining the values of $\beta$, which maximize the log likelihood, while treating the variance parameters as constant. Consequently, the variance parameters are estimated by maximizing the REML log likelihood with regard to the variance. Given the nature of the REML likelihood and its treatment of fixed effects as 
parameters rather than as constants, the resulting variance parameter estimates can be considered as unbiased. Moreover, it reduces the bias inherent in the ML estimates of covariance parameters [14].

\section{Results}

\section{Separated model}

The backward variable selection method was used to select significant variables. At the end, model assumptions were checked and there were the problem of normality and linearity before transformation of variables. The response variables (pulse rate, respiratory rate and weight) were transformed by using a logarithmic function to solve the problem of normality. The linearity assumption failed for the pulse rate model and the respiratory rate model; therefore, to solve this problem, the follow-up time was transformed to the square root. Figures 1-3 indicate that the transformed model satisfies the assumptions of the linear mixed model.

The four different random effect models, starting from the linear regression model (no random effects), have been explored with selected fixed effects. According to Table 2, the random effects model is better than the linear regression model and including the quadratic time as a random effect does not improve the model for all outcomes (pulse rate, respiratory rate and weight). The linear time effect model fits better than only the intercept model for a log of pulse rate and a $\log$ of respiratory rate, whereas the intercept model fits better for a log of weight. 


\section{Table 2 Random effects models}

\begin{tabular}{|c|c|c|c|c|c|}
\hline Where & & The models & AIC & $\mathrm{BIC}$ & $-2 \mathrm{LL}$ \\
\hline $\begin{array}{l}\log P r \\
=\log \end{array}$ & $\log \operatorname{Pr}$ & Without random effects & -3352.2 & -3347.1 & -3354.2 \\
\hline $\begin{array}{c}\text { of } \\
\text { pulse }\end{array}$ & & With only intercept & -3663.9 & -3654.8 & -3669.9 \\
\hline rate, & & With intercept and slope & -3678.3 & -3669.2 & -3684.3 \\
\hline $\begin{array}{l}\log R r \\
=\log \end{array}$ & & Intercept, Linear and Quadratic Slope & -3604.2 & -3595.1 & -3610.2 \\
\hline of & $\log \mathrm{Rr}$ & Without random effects & -2691.6 & -2686.5 & -2693.6 \\
\hline $\begin{array}{l}\text { respir } \\
\text { atory }\end{array}$ & & With only intercept & -3204.1 & -3195.0 & -3210.1 \\
\hline rate & & With intercept and slope & -3319.0 & -3309.9 & -3325.0 \\
\hline $\begin{array}{c}\text { and } \\
\log W h\end{array}$ & & Intercept, Linear and Quadratic Slope & -3149.8 & -3140.7 & -3155.8 \\
\hline $\begin{array}{c}=\log \\
\text { of }\end{array}$ & $\log \mathrm{Wh}$ & Without random effects & -3339.5 & -3334.4 & -3341.5 \\
\hline weight & & With only intercept & -6014.5 & -6005.4 & -6020.5 \\
\hline $\mathrm{F}$ & & With intercept and slope & -5676.7 & -5667.6 & -5682.7 \\
\hline or $\mathrm{a}$ & & Intercept, Linear and Quadratic Slope & -3928.8 & -3922.7 & -3932.8 \\
\hline
\end{tabular}

random effects model, identifying the appropriate Variance-Covariance structure is necessary. Therefore, models with six Variance-Covariance structures of random effects (Unstructured, Compound Symmetry, Heterogeneous Compound Symmetry, Autoregressive Order One, Toeplitz and Variance Components) were fitted. Table 3 indicates that Unstructured and Heterogeneous Compound Symmetry Covariance structures were appropriate for a random effect of a log of pulse rate and a $\log$ of respiratory rate models. The Variance Components, Unstructured and Toeplitz Covariance structure were appropriate for a random effect of a log of weight model.

Fitting the linear mixed model with only the appropriate variance-covariance structure of random effect may not be sufficient; thus, selecting the appropriate correlation structure of measurement errors may also be necessary. For this reason, models with three correlation structures 
of measurement errors (compound symmetry, variance components and autoregressive) were fitted. According to Table 4, for all outcomes, the autoregressive correlation structure was appropriate to model the dependency of the observations within congestive heart failure patients.

Table 3 Variance-covariance structure of random effects

\begin{tabular}{|c|c|c|c|c|}
\hline & Model & AIC & BIC & -2res- LL \\
\hline \multirow{6}{*}{$\begin{array}{l}\stackrel{7}{00} \\
0\end{array}$} & Compound symmetry & -3678.3 & -3669.2 & -3684.3 \\
\hline & Heterogeneous compound symmetry & -3810.0 & -3797.9 & -3818.0 \\
\hline & Unstructured & -3810.0 & -3797.9 & -3818.0 \\
\hline & Autoregressive & -3678.3 & -3669.2 & -3684.3 \\
\hline & Toeplitz & -3678.3 & -3669.2 & -3684.3 \\
\hline & Variance components & -3711.1 & -3702.0 & -3717.1 \\
\hline \multirow{6}{*}{$\begin{array}{l}\overrightarrow{0} \\
\frac{0}{00} \\
\stackrel{0}{1}\end{array}$} & Compound symmetry & -3319.0 & -3309.9 & -3325.0 \\
\hline & Heterogeneous compound symmetry & -3504.1 & -3492.0 & -3512.1 \\
\hline & Unstructured & -3504.1 & -3492.0 & -3512.1 \\
\hline & Autoregressive & -3319.0 & -3309.9 & -3325.0 \\
\hline & Toeplitz & -3319.0 & -3309.9 & -3325.0 \\
\hline & Variance components & -3367.2 & -3358.1 & -3373.2 \\
\hline \multirow{6}{*}{$\sum_{0=0}^{5}$} & Compound symmetry & -6014.5 & -6005.4 & -6020.5 \\
\hline & Heterogeneous compound symmetry & -6014.5 & -6005.4 & -6020.5 \\
\hline & Unstructured & -6016.5 & -6010.5 & -6020.5 \\
\hline & Autoregressive & -6014.5 & -6005.4 & -6020.5 \\
\hline & Toeplitz & -6016.5 & -6010.5 & -6020.5 \\
\hline & Variance components & -6016.5 & -6010.5 & -6020.5 \\
\hline
\end{tabular}

Where $\log P r=\log$ of pulse rate, $\log R r=\log$ of respiratory rate and $\log W h=\log$ of weight

Table 4 Correlation structure of measurement errors for separated models

\begin{tabular}{lllll} 
& Models & AIC & BIC & $-2 \mathrm{LL}$ \\
$\log \operatorname{Pr}$ & Compound symmetry & -3808.0 & -3792.8 & -3818.0 \\
& Variance components & -3810.0 & -3797.9 & -3818.0 \\
& Autoregressive & -3865.6 & -3850.4 & -3875.6 \\
\hline $\operatorname{LogRr}$ & Compound symmetry & -3502.1 & -3486.9 & -3512.1
\end{tabular}




\begin{tabular}{lllll} 
& Variance components & -3504.1 & -3492.0 & -3512.1 \\
& Autoregressive & -3578.9 & -3563.7 & -3588.9 \\
\hline \multirow{2}{*}{ LogWh } & Compound symmetry & -6014.5 & -6005.4 & -6020.5 \\
& Variance components & -6016.5 & -6010.5 & -6020.5 \\
& Autoregressive & -6311.2 & -6302.1 & -6317.2
\end{tabular}

\section{Bivariate model}

There were three joint bivariate models in this study for each pair of outcomes (pulse rate and respiratory rate, respiratory rate and weight and (pulse rate and weight). They were $\log \operatorname{Pr}$ (log pulse rate), $\log R r(\log$ respiratory rate) and $\log W h(\log$ weight). In the same way as the separated models, they were fitted with six variance-covariance structures of random effects (compound symmetry, heterogeneous compound symmetry, unstructured, autoregressive, Toeplitz and variance components). Table 5 shows that, among the fitted covariance structure models, the unstructured covariance structure was the appropriate covariance structure of random effects for all joint bivariate models. In addition to modelling with appropriate variance-covariance structures of random effects, the three-correlation structure was used to model the measurement errors (compound symmetry, autoregressive order one and variance components) was used to model the measurement errors). According to Table 6, the most appropriate correlation structures of error is Autoregressive Order One for all bivariate models, since a model with this correlation structure has the smallest AIC values, BIC values, and Log Likelihood scores compared to the other model.

\section{Table 5 Variance-covariance structures of random effects for bivariate models}

\begin{tabular}{ll} 
& Model \\
& Compound symmetry \\
& Heterogeneous compound symmetry \\
& Variance components \\
& Unstructured \\
\hline 0 & Autoregressive
\end{tabular}

$\begin{array}{lll}\text { AIC } & \text { BIC } & -2 \mathrm{LL} \\ -6873.6 & -6864.5 & -6879.6 \\ -7095.3 & -7077.1 & -7085.5 \\ -7075.9 & -7060.7 & -7085.9 \\ -7403.4 & -7370.0 & -7425.4 \\ -6897.5 & -6888.4 & -6903.5\end{array}$




\begin{tabular}{|c|c|c|c|c|}
\hline & Toeplitz & -6964.6 & -6949.4 & -6974.6 \\
\hline \multirow{6}{*}{$\begin{array}{l}\sum_{00}^{0} \\
0 \\
0 \\
\stackrel{0}{0.0} \\
0 \\
0\end{array}$} & Compound symmetry & -8558.0 & -8482.1 & -8608.0 \\
\hline & Heterogeneous compound symmetry & -8693.1 & -8611.1 & -8747.1 \\
\hline & Variance components & -8639.8 & -8560.9 & -8691.8 \\
\hline & Unstructured & -8816.6 & -8728.5 & -8874.6 \\
\hline & Autoregressive & -8522.4 & -8446.5 & -8572.4 \\
\hline & Toeplitz & -8579.4 & -8500.5 & -8631.4 \\
\hline \multirow{6}{*}{$\begin{array}{l}\sum_{00}^{5} \\
0 \\
0 \\
0 \\
0100 \\
0 \\
0\end{array}$} & Compound symmetry & -8747.1 & -8737.9 & -8753.1 \\
\hline & Heterogeneous compound symmetry & -8852.3 & -8837.1 & -8862.3 \\
\hline & Variance components & -8811.8 & -8799.7 & -8819.8 \\
\hline & Unstructured & -8977.4 & -8956.2 & -8991.4 \\
\hline & Autoregressive order one & -8722.6 & -8713.5 & -8728.6 \\
\hline & Toeplitz & -8784.8 & -8772.7 & -8792.8 \\
\hline
\end{tabular}

Table 6 Correlation structures of measurement errors for bivariate models

\begin{tabular}{|c|c|c|c|c|}
\hline & Model & AIC & $\mathrm{BIC}$ & $-2 \mathrm{LL}$ \\
\hline \multirow{3}{*}{$\begin{array}{l}\overrightarrow{0} \\
0 \\
0 \\
0\end{array}$} & Compound symmetry & -7401.4 & -7365.0 & -7425.4 \\
\hline & Variance components & -7403.9 & -7367.4 & -7427.9 \\
\hline & Autoregressive & -7523.9 & -7481.3 & -7551.9 \\
\hline \multirow{3}{*}{$\begin{array}{l}3 \\
000 \\
0 \\
0.0 \\
00 \\
0 \\
0\end{array}$} & Compound symmetry & -8814.6 & -8723.5 & -8874.6 \\
\hline & Variance components & -9774.4 & -9683.3 & -9834.4 \\
\hline & Autoregressive & -10178.7 & -10081.6 & -10242.7 \\
\hline \multirow{3}{*}{ 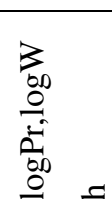 } & Compound symmetry & $\begin{array}{l}-8989.0 \\
\end{array}$ & -8964.7 & -9005.0 \\
\hline & Variance components & -9884.3 & -9860.0 & -9900.3 \\
\hline & Autoregressive & -10242.6 & -10212.3 & -10262.6 \\
\hline
\end{tabular}




\section{Multivariate model}

The multivariate model employed in this study aim at the joint modeling of the three outcomes (PR, RR, Wh). Similar to the aforementioned models, it was fitted with six different variancecovariance structures (compound symmetry, heterogeneous compound symmetry, unstructured, autoregressive, Toeplitz and variance components). Table 7 indicates that among the six variancecovariance structures of random effects, the unstructured one is most appropriate for the joint multivariate model. However, modeling with only an appropriate variance-covariance structure may not fully guarantee the construction of a good model. Therefore, the multivariate model has to be fitted again with the three correlation structures (i.e. compound symmetry, autoregressive and variance components) of the measurement errors. According to Table 8, a model with autoregressive order-one correlation structures can most efficiently model the correlation structures of the outcome (PR, RR, Wh) measurements concerning congestive heart failure patients.

Table 7 Variance covariance structures of random effects for multivariate model

\begin{tabular}{llll} 
Multivariate model & AIC & BIC & $-2 \mathrm{LL}$ \\
\hline Compound symmetry & -11838.7 & -11829.6 & -11844.7 \\
Heterogeneous compound symmetry & -12083.3 & -12062.0 & -12097.3 \\
Unstructured & -12466.8 & -12418.2 & -12498.8 \\
Autoregressive & -11857.2 & -11848.1 & -11863.2 \\
Toeplitz & -11969.1 & -11950.8 & -11981.1 \\
Variance components & -12053.2 & -12035.0 & -12065.2
\end{tabular}

Table 8. Correlation structures of measurement errors for multivariate model

\begin{tabular}{llll}
\hline Multivariate model & AIC & BIC & $-2 \mathrm{LL}$ \\
\hline Compound symmetry & -12464.8 & -12413.2 & -12498.8 \\
Variance components & -13514.4 & -13459.7 & -13550.4 \\
Autoregressive & -13959.2 & -13895.4 & -14001.2 \\
\hline
\end{tabular}




\section{Discussion}

In this study three models were considered for fitting three outcome variables of congestive heart failure patients which measured longitudinally. They were separate, bivariate and multivariate linear mixed effects models. The models contain the fixed and random effect components. The appropriate fixed effects and random effects were selected by using the maximum likelihood estimation method and restricted maximum likelihood estimation method respectively. The linear mixed model without random effects, with only intercept, with intercept and linear time, and with intercept and quadratic time were fitted. The fitted random effect models were compared for the purpose of selecting the best random effects that enable to account the variability between congestive heart failure patients as $[12,15]$. The four random effect models were compared using the AIC, BIC and -2LL value. Though a model with random effects intercept and linear time was the best after transformation of time for a $\log$ of pulse rate and $\log$ of respiratory rate in to the square root of time and the response variable pulse rate, respiratory rate and weight into logarithmic function which contradict with [12] in which time was linear and the distributions were normal without transformation.

The covariance structures of random effects which used in this study were; unstructured, compound symmetry, heterogeneous compound symmetry, Toeplitz, variance components and autoregressive covariance structure of order one. In similar to the study [7, 12, 15, and 16] the unstructured covariance structure was the most appropriate covariance structure of the models from the random effects of separation, bivariate and multivariate models.

In order to model dependence among observations the correlation structure of the measurement error was considered. The correlation structures which used in this study were compound symmetric; variance components and autoregressive correlation structure of order one 
and compared to select the most appropriate one by using AIC, BIC and -2LL. The autoregressive structure of order one was the most appropriate correlation structure of the measurement error as [17, 18] incorporated into mixed models to consider the dependence among the observations which contradict the independence assumptions in $[12,15,16]$. The congestive heart failure is chronic disease which does not recover in short time. The measurement of the outcome of the congestive heart failure patients does not decrease very quickly. Taking the autoregressive structure of order one correlation structure of the measurement error is appropriate as [13] stated that; the assumption of independent correlation may not hold if the observation does not decrease very quickly.

Finally, with the selected covariance structure of random effects and correlation structure of measurement errors, the three models (separate, bivariate and multivariate models) were compared by using AIC, BIC and -2LL. The multivariate model is better than bivariate model is better than separate model. It shows that for congestive heart failure data joint model is better than separate as $[7,12,15$, and 19].

\section{Conclusion}

For congestive heart failure data the random effect model is better with unstructured covariance structure of random effects and an Auto-regressive order one correlation structure of measurement error. Some studies consider the only covariance structure of random effects, in addition to the covariance structure of random effects considering the correlation structure of repeated effects needed for congestive heart failure data. Though using independent correlation structures of measurement error model may display less accurate results.

Three models considered in this study were separate, bivariate and multivariate for fitting three response variables measured longitudinally. The multivariate model with an unstructured covariance structure of random effects and an Auto-regressive order one correlation structure of measurement 
error is the best model compared to the separate and joint bivariate models because its standard error of the parameter estimates is smaller. And also, the multivariate model has a very small AIC value which indicates that it fits the data better than the separate and joint bivariate models.

In this study, it focuses on only three response variables with correlation structure of measurement errors which were compound symmetry, variance components and autoregressive order one, for future work, one might want to look at modeling more than three response variables over time with an additional correlation structure of the measurement error.

\section{Abbreviations}

ACF: Acute Coronary failure, AIC: Akaike Information Criterion, AU: Africa Union, BIC: Bayesian Information Criterion, CAD: Coronary artery disease, CHD: Coronary heart disease, CHF: Congestive heart failure, GEE: Generalized estimating equations, GLM: Generalized Linear Model, Hr: Heart rate, HF: Heart failure, ICC: Intra-class Correlation Coefficient, LMM: Linear mixed model, LVEF: Left-ventricular ejection fraction, MLIRT: Multilevel item response theory, MLMM: Multivariate linear mixed model, MQL: Marginal Quasi-Likelihood, NYHAC: New York heart association class, PQL: Penalized Quasi-Likelihood, Pr: Pulse rate, Rr: Respiration rate, SBP: Systolic blood pressure, DBP: Diastolic blood pressure, Sqrtim: square root of time, Wh: Weight

\section{Authors' contributions}

MS conceptualized the idea conceive, designed, wrote the study, participated in data collection, analyzed the data, interpreted the result, participated in the drafting the manuscript and approved the final manuscript for submission. RA, participated in the drafting the manuscript, revised the manuscript and approved the final manuscript for submission, responsible for submission and communication about the manuscript. 


\section{Funding}

This research was funded by the Jimma University. The funding body had no role in the design or analysis of the study, interpretation of results, or writing of the manuscript.

\section{Availability of data and materials}

All relevant data are within the manuscript and its supporting information files.

\section{Ethics approval and consent to participate}

The ethics approval was obtained from Jimma University review board (IRB). Additionally, permission was obtained from the hospital authority before beginning the data collection.

\section{Consent for publication}

Not applicable.

\section{Competing interests}

The authors declare that they have no competing interests.

\section{Acknowledgements}

We extend our gratitude to Jimma University, Hospital authorities, supervisors, data collectors, and respondents who participated on this study. 


\section{References}

1. Wang KS. Linear and Non-Linear Mixed Models in Longitudinal Studies and Complex Survey Data. J Biom Biostat. 2016; 7: 290. doi:10.4172/2155-6180.1000290.

2. Yende N. Modelling CD4+ Count Over Time in HIV Positive Patients Initiated on HAART in South Africa Using Linear Mixed Models. MSC. Thesis, the University of KwaZulu Natal,

December $2009 . \quad$ Available at: https://pdfs.semanticscholar.org/db70/3bf91673ce2782f09d52f776542550f0e40f.pdf

3. Ene M, Leighton EA, Blue GL, Bell BA. Multilevel Models for Categorical Data Using SAS®PROC: The Basics. Paper 3430-2015, 2015.

4. Verbeke G, Fieuws S, Molenberghs G, Davidian $\mathrm{M}$. The analysis of multivariate longitudinal data: a review. Stat Methods Med Res. 2014; 23(1): 42-59.

5. Sammel M, Lin X, Ryan L. Multivariate linear mixed models for multiple outcomes. Stat Med. 1999; 18:2479-2492.

6. Santos AA, Nogales FJ, Ruiz E. Comparing univariate and multivariate models to forecast portfolio value-at-risk. Journal of financial econometrics. 2013; 11(2):400-41.

7. Thiébaut R, Jacqmin-Gadda H, Chêne G, Leport C, Commenges D. Bivariate Linear Mixed Models Using SAS Proc MIXED, Comput Methods Programs Biomed. 2002 ;69(3):249-56.

8. Badiru AB. Computational survey of univariate and multivariate learning curve models. Engineering Management, IEEE Transactions on. 1992; 39(2):176-88.

9. Santos AA, Nogales FJ, Ruiz E. Comparing univariate and multivariate models to forecast portfolio value-at-risk. Journal of financial econometrics. 2013; 11(2):400-41.

10. Edwards LJ. Modern statistical techniques for the analysis of longitudinal data in biomedical research. Pediatric Pulmonology. 2000; 30(4):330-344. 
11. Pinheiro JC and Bates DM. Mixed-Effects Models in S and S-PLUS. Springer Verlag New York, 2000; 96.

12. Olkin, I. and Tate, R. Multivariate correlation models with mixed discrete and continuous variables. Ann of Math Stat. 1961; 32, 448-465.

13. Fissuh and Muleta. A Joint Model for a Longitudinal Pulse Rate and Respiratory Rate of Congestive Heart Failure Patients: at Ayder Referral Hospital of Mekelle University, Tigray, Ethiopia. J Biom Biostat. 2015; 6:5.,DOI: 10.4172/2155-6180.1000260.

14. Browne W, Goldstein H. MCMC sampling for multilevel model with non-independent residuals within and between cluster units. Journal of Educational and Behavioral Statistics. 2010; 35(4), 453-473. Doi: 10.3102/1076998609359788.

15. Morrell, C.H. Likelihood ratio testing of variance components in the linear mixed-effects model using restricted maximum likelihood. Biometrics 1998; 54, 1560.

16. Negash Y, Kassahunb W , Gurmessaa A and Birliea B. Joint modeling of longitudinal systolic and diastolic blood pressure measurements of hyper-tensive patients receiving treatment. Electronic Journal of Applied Statistical Analysis EJASA, Electron. J. App. Stat. Anal 2016; 09(02): 308-325.

17. Demeke L, Dereje T, Haile M. Bivariate longitudinal data analysis: a case of hypertensive patients at Felege Hiwot Referral Hospital, Bahir Dar, Ethiopia. BMC Res. 2017; 10:722.

18. He B and Luo S. Joint modeling of multivariate longitudinal measurements and survival data with application to Parkinson's disease. Stat Methods Med Res. 2016; 25(4): 1346-1358. doi:10.1177/0962280213480877.

19. Chi, E. M. and Reinsel, G. C. Models for longitudinal data with random effects and AR (1) errors. Journal of the American Statistical Association. 1989; 84: 452-459. 56. 
20. Lindstrom, M. J. and Bates, D. M. Nonlinear mixed effects models for repeated measures data. Biometrics. 1990; 46: 673-687. 


\section{Supplementary Files}

This is a list of supplementary files associated with this preprint. Click to download.

- suportive.docx 